\title{
Novel Data about Association of the Functionally Significant Polymorphisms of the MMP9 Gene with Exfoliation Glaucoma in the Caucasian Population of Central Russia
}

\author{
Dina Starikova $^{\mathrm{a}}$ Irina Ponomarenko ${ }^{\mathrm{a}}$ Evgeny Reshetnikov ${ }^{\mathrm{a}}$ \\ Volodymyr Dvornyk ${ }^{b}$ Mikhail Churnosov ${ }^{a}$ \\ aDepartment of Medical Biological Disciplines, Belgorod State University, Belgorod, Russia; ${ }^{b}$ Department of Life \\ Sciences, College of Science and General Studies, Alfaisal University, Riyadh, Saudi Arabia
}

\section{Keywords}

Association - Matrix metalloproteinases - Exfoliation

glaucoma $\cdot$ Single-nucleotide polymorphism

\begin{abstract}
Aim: This study aimed to investigate the role of functionally significant polymorphisms of the MMP1, MMP3, and MMP9 genes in the development of exfoliation glaucoma (XFG) in the Caucasian population of Central Russia. Methods: The study sample consisted of 724 participants, including 328 patients with XFG and 396 individuals in the control group. The participants were of Russian ethnicity (self-reported) born in Central Russia. The participants were genotyped at 8 functionally significant polymorphisms of the MMP genes (rs3918242, rs3918249, rs17576, rs3787268, rs2250889, rs17577 MMP9, rs679620 MMP3, and rs1799750 MMP1). The association analysis was performed using logistic regression. Two polymorphisms, which were associated with XFG, and 12 polymorphisms linked to them $\left(r^{2} \geq 0.8\right)$ were analyzed for
\end{abstract}

their functional significance in silico. Results: Allele $C$ of rs3918249 MMP9 was associated with XFG according to the additive model ( $\left.\mathrm{OR}=0.75,95 \% \mathrm{Cl}: 0.56-0.93, p_{\text {perm }}=0.015\right)$, and allele $\mathrm{G}$ of the rs2250889 MMP9 locus was associated with XFG according to the additive $(\mathrm{OR}=1.59,95 \% \mathrm{Cl}: 1.10$ $\left.2.29, p_{\text {perm }}=0.013\right)$ and dominant $(\mathrm{OR}=1.68,95 \% \mathrm{Cl}: 1.11-$ $\left.2.56, p_{\text {perm }}=0.016\right)$ models. Two XFG-associated loci of the MMP9 gene and 12 SNPs linked to them had a significant regulatory potential (they are located in the evolutionarily conserved regions, promoter and enhancer histone marks, the DNAase-hypersensitivity regions, a region binding to regulatory protein, and a region of regulatory motifs) and may influence the expression of 13 genes and alternative splicing of 4 genes in various tissues and organs related to the pathogenesis of XFG. Conclusion: Allele C rs3918249 MMP9 decreased risk for XFG $(\mathrm{OR}=0.75)$, and allele $\mathrm{G}$ of the rs2250889 MMP9 locus increased risk for XFG (OR $=1.59$ 1.68) in the Caucasian population of Central Russia.

(c) 2020 S. Karger AG, Basel karger@karger.com www.karger.com/ore

(c) 2020 S. Karger AG, Base

Karger! $\stackrel{\text { ' }}{=}$
Evgeny Reshetnikov

Department of Medical Biological Disciplines, Belgorod State University 85 Pobedy St.

Belgorod 308015 (Russian Federation)

reshetnikov@bsu.edu.ru 


\section{Introduction}

Glaucoma is recognized as one of the principal causes of irreversible blindness worldwide [1]. It is a group of heterogeneous neurodegenerative diseases with a common pathogenic pathway, namely, progressive loss of retinal ganglion cells (RGCs) and optic nerve axons resulting in visual field defects [2]. Within this diverse group of diseases, exfoliation glaucoma (XFG) is a frequent and progressive subtype accounting for $25-70 \%$ of open-angle glaucoma and conferring a high risk of blindness [3]. $\mathrm{XFG}$ is characterized by rapid progression, high resistance to medical therapy, and poor prognosis [4].

$\mathrm{XFG}$ is thought to develop due to the obstruction of the aqueous humor outflow, which is caused by the deposition of extracellular protein aggregates and subsequent increase in intraocular pressure and ultimately glaucomatous optic nerve damage. This abnormal accumulation of the extracellular matrix (ECM) occurs in the course of a common age-related, generalized disorder termed exfoliation syndrome, which affects up to $30 \%$ of seniors [5].

A significant role in the synthesis and degradation of the ECM, including the ECM of the trabecular meshwork of the eye, belongs to matrix metalloproteinases (MMPs) [6]. MMPs are a group of zinc- and calcium-dependent endopeptidases that are involved in ECM homeostasis and remodeling [7]. Most importantly, MMPs are responsible for regulating the outflow of aqueous humor from the anterior chamber of the eye and therefore have a significant impact on intraocular pressure $[8,9]$. A significantly altered level of MMPs in the aqueous humor of patients with diagnosed XFG was previously described [8, 10]. The elevated expression of MMP1 was documented in the optic nerve heads of both human glaucomatous eyes and monkey eyes with experimental glaucoma [11, 12]. Furthermore, ligation of the optic nerve caused an increase in the MMP9 expression in the mouse retina resulting in apoptotic ganglion cell loss [13].

Despite the apparent contribution of MMP to the development of XFG, the studies on possible association of the $M M P$ polymorphisms with XFG are limited. We have been able to find only 4 reports, 2 of which were experimental $[14,15]$ and the other 2 were meta-analyses based on these experimental works $[16,17]$. Tsironi et al. [14] did not determine the significant association of 2 SNPs of $M M P 1$ and $M M P 3$ with XFG in the sample of 92 patients and 214 controls from Greece. Likewise, no significant association with the disease was found for the SNPs of $M M P 1, M M P 2$, and $M M P 9$ in the study sample with 202 patients and 248 controls from Austria [15]. The results of the abovementioned meta-analyses are contradictory. $\mathrm{Wu}$ et al. [16] did not find a significant association of rs1799750 MMP1 and rs17576 MMP9 with XFG, whereas He et al. [17] reported such association for rs 1799750 $M M P 1$. It is worth noting, however, that the result reported by Wu et al. [16] is, in fact, just a restatement of the result from the original work of Mossböck et al. [15].

The obvious lack of experimental studies on the possible contribution of the $M M P$ gene polymorphisms to XFG prompts for filling this gap. This study was aimed to analyze the role of functionally significant polymorphisms of $M M P 1, M M P 3$, and $M M P 9$ genes in the development of XFG in the sample of Caucasians from Central Russia.

\section{Materials and Methods}

\section{Study Subjects}

The study sample consisted of 724 participants, including 328 patients with XFG and 396 controls. When recruiting the participants, the following inclusion criteria were applied: Russian ethnicity (self-reported) and birthplace in Central Russia [18]. XFG was diagnosed based on (a) the presence of exfoliation material on the lens capsule, iris, or corneal endothelium and (b) the presence of glaucomatous optic disc cupping, visual field loss, and IOP $\geq 21$ $\mathrm{mm} \mathrm{Hg}$ or controlled IOP on antiglaucomatous treatment in at least one eye. Pupillary dilation was performed on all participants to determine the presence of exfoliation material on the anterior lens capsule. Control subjects had no signs of exfoliation deposits on anterior segment structures, repeated IOP readings of $<21 \mathrm{~mm}$ $\mathrm{Hg}$, and normal optic discs.

All study participants signed written informed consent per the principles of the Helsinki Declaration before enrollment in the study. The medical examination of the participants was conducted at the Department of Eye Microsurgery of St. Iasaf Belgorod Regional Clinical Hospital.

\section{DNA Isolation and Genotyping Assay}

Approximately 4-5 mL blood sample from each participant was collected in vacutainer tubes (Vacutainer $\left.{ }^{\circledR}\right)$. DNA was extracted from whole blood by the phenol-chloroform method and then checked for quality (as described earlier by Ponomarenko et al. [19]).

Eight SNPs of the 3 MMP genes (rs3918242, rs3918249, rs17576, rs3787268, rs2250889, rs17577 MMP9, rs679620 MMP3, and rs1799750 MMP1) were selected for the analysis. The following selection criteria were applied [20]: (1) previously reported associations with glaucoma (XFG) (see online suppl. Table 1; see www. karger.com/doi/10.1159/000512507 for all online suppl. material), (2) regulatory potential (online suppl. Table 2), and (3) MAF $>5 \%$.

All the above SNPs have a significant regulatory potential (determined using the online tools HaploReg, v4.1 update November 5, 2015, https://pubs.broadinstitute.org/mammals/haploreg/haploreg.php) (online suppl. Table 2), 7 of them were reported for the association with glaucoma (including 2 SNPs associated with 
Table 1. Phenotypic characteristics of the study participants

\begin{tabular}{|c|c|c|c|}
\hline Parameters & $\begin{array}{l}\text { Controls, } \\
\text { mean } \pm \text { SD, \% (n) }\end{array}$ & $\begin{array}{l}\text { XFG patients, } \\
\text { mean } \pm \mathrm{SD}, \%(n)\end{array}$ & $p$ value \\
\hline$N$ & 396 & 328 & - \\
\hline Age, years (min-max) & $62.02 \pm 11.54(42-87)$ & $71.64 \pm 8.67(43-94)$ & $<0.001$ \\
\hline Women & $55.56(220)$ & $52.74(173)$ & 0.49 \\
\hline $\mathrm{BMI}, \mathrm{kg} / \mathrm{m}^{2}$ & $27.95 \pm 5.45$ & $27.57 \pm 4.69$ & 0.53 \\
\hline Mean systolic blood pressure, mm Hg & $130.87 \pm 14.83$ & $142.29 \pm 17.67$ & $<0.001$ \\
\hline Mean diastolic blood pressure, $\mathrm{mm} \mathrm{Hg}$ & $84.08 \pm 9.57$ & $87.24 \pm 11.57$ & $<0.001$ \\
\hline Smoke & $28.03(111)$ & $25.91(85)$ & 0.58 \\
\hline Alcohol & $32.07(127)$ & $30.18(99)$ & 0.64 \\
\hline Family history of glaucoma & $6.06(24)$ & $20.12(66)$ & $<0.001$ \\
\hline \multicolumn{4}{|l|}{ Ophthalmological characteristics } \\
\hline Intraocular pressure, $\mathrm{mm} \mathrm{Hg}$ & $16.41 \pm 1.54$ & $25.19 \pm 5.78$ & $<0.001$ \\
\hline Cup to disc ratio & $0.25 \pm 0.08$ & $0.72 \pm 0.31$ & $<0.001$ \\
\hline \multicolumn{4}{|l|}{ Somatic pathologies } \\
\hline Essential hypertension & $61.11(242)$ & $72.56(238)$ & 0.002 \\
\hline Arterial hypotension & $5.81(23)$ & $4.27(14)$ & 0.44 \\
\hline Heart atherosclerosis & $14.14(56)$ & $29.57(97)$ & $<0.001$ \\
\hline Heart ischemia & $24.00(95)$ & $35.36(116)$ & $<0.001$ \\
\hline Diabetes & $10.10(40)$ & $15.24(50)$ & 0.05 \\
\hline Digestive system pathology & $12.88(51)$ & $14.33(47)$ & 0.65 \\
\hline Kidney pathology & $7.32(29)$ & $8.54(28)$ & 0.64 \\
\hline Respiratory system pathology & $5.05(20)$ & $6.10(20)$ & 0.65 \\
\hline Nervous system pathology & $9.09(36)$ & $9.45(31)$ & 0.97 \\
\hline
\end{tabular}

Statistically significant $p$ value is given in bold. XFG, exfoliation glaucoma.

XFG) (online suppl. Table 1), and one SNP (rs679620 MMP3), although being not associated with glaucoma per se, was associated with the vascular risk factors of the XFG (blood pressure, hypertension, and atherosclerosis) [21,22].

Genotyping for all the polymorphisms was done using the MALDI-TOF mass spectrometry iPLEX platform (Agena Bioscience Inc., San Diego, CA, USA). Genotyping blind replicates were included to ensure quality control. The repeatability test was conducted for $5 \%$ randomly selected samples and yielded $100 \%$ reproducibility.

\section{Statistical Analysis}

The Hardy-Weinberg equilibrium of the determined allele and genotype frequencies was estimated by the $\chi^{2}$ test. The logistic regression method was applied to analyze associations of the SNPs with XFG assuming additive (i.e., comparison of all genotypes, e.g., GG vs. GA vs. AA), recessive (AA vs. GA/GG, where $A$ is a minor allele), and dominant (GG/GA vs. AA, where $A$ is a minor allele) genetic models with adjustment for covariates. Age, systolic blood pressure, and diastolic blood pressure were used as quantitative variables (value of the trait), whereas family history of glaucoma and the presence of essential hypertension, heart atherosclerosis, heart ischemia, and diabetes mellitus (either type I or type II) as qualitative variables (yes/no) (Table 1). Adjustment for multiple comparisons was performed using the adaptive permutation test [23]. The significance level was set at $p_{\text {perm }}<0.017$ (after the Bonferroni correction based on the numbers of genetic models stud- ied, $n=3)$. Statistical power for each SNP was computed using Quanto 1.2.4 (http://hydra.usc.edu/gxe 2009). The haplotype blocks were identified using the "confidence intervals" algorithm at $\mathrm{D}^{\prime}>0.8$ as implemented in HaploView v.4.2 (https://www. broadinstitute.org/haploview/haploview). The association analyses and adaptive permutation test were performed using the respective algorithms implemented in the PLINK v. 2.050 software (http://zzz.bwh.harvard.edu/plink/).

\section{Functional SNPs}

The genetic variants associated with XFG and SNPs, which were strongly linked to them, were evaluated for their functional significance (nonsynonymous SNPs, regulatory potential, eQTLs, and sQTLs). SNPs in strong linkage disequilibrium (LD) $\left(r^{2} \geq 0.8\right)$ with the XFG-associated ones were identified using HaploReg (v4.1) (http://archive.broadinstitute.org/mammals/haploreg/haploreg.php). The LD was estimated using the data of the European population from the 1,000 Genomes Project Phase. The SIFT online tool (http://sift.jcvi.org/) and PolyPhen-2 online tool (http:// genetics.bwh.harvard.edu/pph2/index.shtml) were used to analyze nonsynonymous SNPs and their functional predictions.

The regulatory potential of the candidate SNPs for XFG was analyzed in silico using HaploReg (v4.1). The affinity between the factor-binding site of the reference (ref) and alternative (alt) alleles of a locus and transcription factors was estimated as a difference between the LOD scores of the alleles [24]: $\triangle \mathrm{LOD}=\mathrm{LOD}$ (alt) LOD (ref). A negative value indicates the increased affinity of this 
Table 2. Associations of the MMP gene polymorphisms with XFG

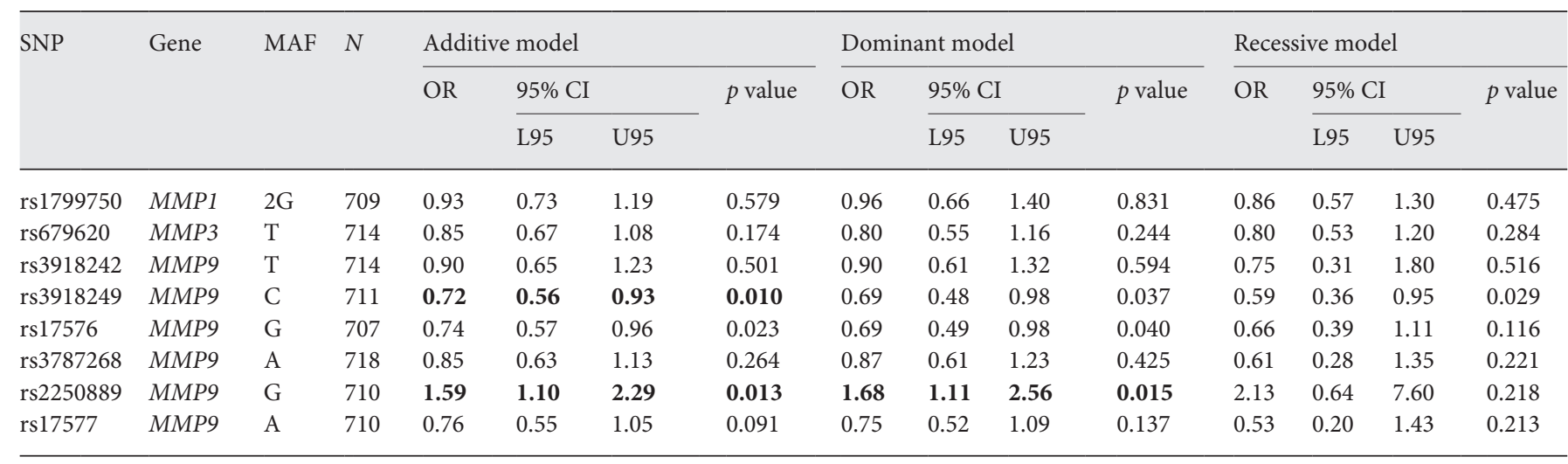

All results were obtained after adjustment for covariates. XFG, exfoliation glaucoma; OR, odds ratio; 95\% CI, 95\% confidence interval. Bold values denote statistical significance at the $p<0.05$ level.

motif by the reference allele, while a positive value suggests the respective increase for the alternative allele.

GTExportal data (http://www.gtexportal.org/) as of December 10, 2017 (release V7 updated on May 9, 2017) (dbGaP Accession phs000424.v7.p2) were used to estimate an effect of the candidate SNPs for XFG on gene expression (cis- and trans-eQTL) and splicing QTLs. The false discovery rate (FDR) $\leq 0.05$ was applied to determine significant eQTLs.

\section{Results}

The phenotypic characteristics of the case and control groups are presented in Table 1 . The XFG patients were 9.62 years older $(p<0.001)$ and had higher systolic and diastolic blood pressure $(p<0.001)$, higher rates of family history of glaucoma $(p<0.001)$, the presence of essential hypertension $(p<0.001)$, heart atherosclerosis $(p<$ $0.001)$, heart ischemia $(p<0.001)$, and diabetes $(p=0.05)$ as compared to the controls (Table 1). Therefore, these factors were applied as covariates in the association analyses.

The data about the analyzed SNPs are shown in online suppl. Table 3. All polymorphisms had MAF $>5 \%$ and were in the HWE. Allele C of rs3918249 MMP9 was associated with XFG according to the additive model $\left(\mathrm{OR}=0.75,95 \%\right.$ CI: $0.56-0.93, p=0.010, p_{\text {perm }}=0.015$, power: $84.38 \%$ ), and allele $\mathrm{G}$ of the rs2250889 MMP9 locus was associated with XFG according to the additive $\left(\mathrm{OR}=1.59,95 \%\right.$ CI: $1.10-2.29, p=0.013, p_{\text {perm }}=0.013$, power: $85.13 \%)$ and dominant $(\mathrm{OR}=1.68,95 \% \mathrm{CI}: 1.11-$ $2.56, p=0.015, p_{\text {perm }}=0.016$, power: $\left.85.67 \%\right)$ models $(\mathrm{Ta}$ ble 2). None of the $M M P 9$ gene haplotypes was associated with XFG (Fig. 1, online suppl. Table 4).

Functionally Significant Polymorphisms of $M M P 9$ and Exfoliation Glaucoma

\section{Functional SNP}

One of the 2 XFG-associated SNPs of the MMP9 gene, rs2250889, was missense. It results in the Arg574Pro substitution in the respective protein. This replacement has SIFT score $=1.00$ that corresponds to the predictive value "tolerated" and PolyPhen-2 score $=0.00$ (sensitivity: 1.00 and specificity: 0.00 ) that corresponds to the predictive value "benign." Another XFG-associated polymorphism, rs3918249, is in strong LD with rs17576, which results in an amino acid substitution Gln279Arg in the MMP9 protein (SIFT score $=0.288$ and predictive value "tolerated"; PolyPhen-2 score $=0.004$, sensitivity: 1.00 , specificity: 0.00 , and predictive value "benign").

The results of the HaploReg (v4.1) analysis suggest that both XFG-associated SNPs of the MMP9 gene have a significant regulatory potential (online suppl. Table 2). The rs3918249 polymorphism locates in a promoter histone mark in 4 tissues and enhancer histone mark in blood cells, the DNAase I hypersensitive region in blood cells, and a region of 4 regulatory motifs; rs 2250889 is located in evolutionarily conserved regions (according to both GERP and SiPhy) that have promoter histone mark in 24 tissues and the DNAase I hypersensitive region in 15 tissues, a region binding to regulatory protein (CTCF), and a region of regulatory motifs (NRSF). Allele C of rs3918249 MMP9 increases affinity to 2 transcription factors (TFs), $\operatorname{Hmx}(\Delta \mathrm{LOD}=1.9)$ and Hoxb8 $(\Delta \mathrm{LOD}=2.9)$, and decreases affinity to the other 2 , Arid3a $(\triangle \mathrm{LOD}=-0.7)$ and Pax-5 ( $\triangle \mathrm{LOD}=-3.9)$; allele $\mathrm{G}$ of rs2250889 decreases affinity to transcription factor NRSF $(\triangle \mathrm{LOD}=-11.4)$.

We also studied 12 SNPs, which were tightly linked to the 2 XFG-associated polymorphisms (online suppl. Table 5). Four SNPs of these were located in an evolutionarily 


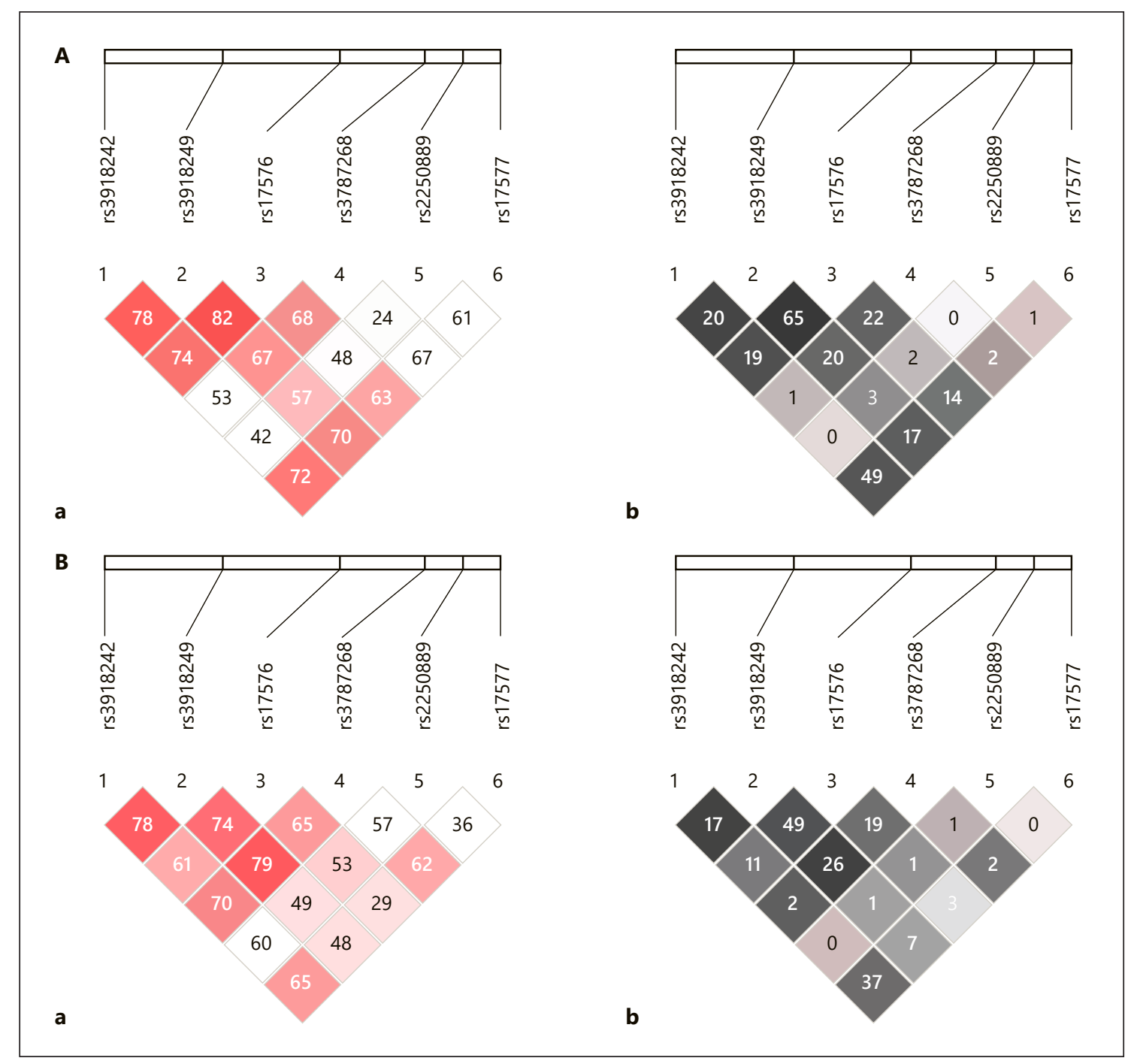

Fig. 1. Linkage disequilibrium (LD) between SNPs rs3918242, rs3918249, rs17576, rs3787268, rs2250889 and rs17577 of the MMP9 gene in XFG patients (A) and control group (B). LD measures are presented as Lewontin's standardized coefficient $\mathrm{D}^{\prime}(\mathbf{a})$ and the square of the correlation Pearson's coefficient $\left(\mathrm{r}^{2}\right)(\mathbf{b})$ between SNPs.

conserved region. Several genetic markers appeared to have significant regulatory potential. For example, rs13969 (linked to rs3918249) is located in an evolutionarily conserved region, a region of histones marking promoters and enhancers in various tissues, the DNAase I hypersensitive region in 24 tissues, a region binding to 7 regulatory proteins (CTCF, RAD21, SMC3, ZEB1, CCNT2, HAE2F1, and ZNF263), and a region of 4 regulatory motifs (ATF3, E2F, $\mathrm{XBP}-1$, and $\mathrm{p} 300$ ). Another polymorphism linked to rs3918249 and rs3848722 manifests similar characteristics, such as being located in an evolutionarily conserved region, region of histones marking promoters and enhancers in various tissues (11 and 14 tissues, respectively), the DNA- ase I hypersensitive region in 19 tissues, and a region of 4 regulatory motifs (HNF4, NRSF, Pax-6, and ZID).

According to the GTExportal database, the XFG-associated SNPs of the MMP9 gene have the cis-eQTL significance $\left(p<8 \times 10^{-5}, p_{\mathrm{FDR}} \leq 0.05\right)$ and may affect the expression of 10 genes in several organs and tissues (online suppl. Table 6). The XFG-associated loci manifested strong linkage to 12 polymorphisms affecting gene expression $\left(p<8.5 \times 10^{-5}, p_{\mathrm{FDR}} \leq 0.05\right)$ of 13 genes in $>20$ organs and tissues, including those pathogenetically significant for the development of XFG (fibroblasts, brain, pituitary, adipose, peripheral blood, and endocrine glands) (online suppl. Table 6). 
Checking against the GTExportal database suggested that the XFG-associated SNPs of the MMP9 gene had sQTL significance $\left(p<8 \times 10^{-5}, p_{\mathrm{FDR}} \leq 0.05\right)$ and might affect alternative splicing of the SLC12A5 gene in the brain (cortex and substantia nigra), pituitary, and the other organs (online suppl. Table 7). These loci manifested strong linkage to 12 polymorphisms, which affect sQTL $\left(p<8.5 \times 10^{-5}, p_{\mathrm{FDR}} \leq 0.05\right)$ of 4 genes $(A C O T 8$, PLTP, SLC12A5, and SNX21) in various tissues and organs (online suppl. Table 7).

\section{Discussion}

We firstly report the results suggesting that polymorphisms rs3918249 and rs2250889 of the MMP9 gene may be the risk factors for XFG: allele C of rs3918249 MMP9 decreases risk for XFG $(\mathrm{OR}=0.75)$, and allele $\mathrm{G}$ of the rs2250889 MMP9 locus increases the risk for the disease $(\mathrm{OR}=1.59-1.68)$ in the Caucasian population of Central Russia. These loci and 12 SNPs linked to them appear to have significant regulatory potential and may affect the expression of 13 genes and alternative splicing of 4 genes in various tissues and organs related to the pathogenesis of XFG.

MMP9 protein plays an important role in ECM remodeling by cleaving denatured collagen and type IV collagen in the basement membrane [7]. Previously, the immediate effect of the high MMP9 expression on the loss of RGCs was reported [13]. The constitutive low expression of MMP9 was observed in the RGC layer [13]. Guo et al. [25] found that the increase of the MMP9 expression in the RG apoptotic cells is associated with the reduced level of laminin, thus indicating the elevated degradation of ECM. A significantly modified level of MMPs in the aqueous humor of patients with XFG was described previously $[8,10]$. Furthermore, optic nerve ligation increased the MMP9 expression in the mouse retina leading to the apoptotic loss of ganglion cells [13].

MMPs have been shown to play an important role in the development of an abnormal ECM in exfoliation syndrome/glaucoma [26]. Increased biosynthesis of MMPs has been found in exfoliation, which may limit the progression of the exfoliation syndrome by degrading the abnormal fibrillar matrix components in the anterior segment tissues; however, it was also suggested that the MMPs might be among factors contributing to exfoliation [27].

Until now, several studies have studied association of the rs3918249 MMP9 polymorphism with development of the primary angle closure glaucoma (PACG) and pri-

Functionally Significant Polymorphisms of $M M P 9$ and Exfoliation Glaucoma mary open-angle glaucoma [28-32], and only one of them reported such association with PACG [28]. Besides, as was mentioned above, possible association of rs3918249 $M M P 9$ with glaucoma was suggested by 2 meta-analyses $[16,33]$.

Locus rs2250889 MMP9, which was determined in the present study as associated with XFG, was previously reported as a candidate for PACG [32, 34]. Interestingly, that allele $\mathrm{G}$ of this locus was determined as a risk factor for both PACG and XFG (OR = 1.57-1.71). On the other hand, no association of this variant with primary openangle glaucoma was determined [32].

In summary, allele $\mathrm{C}$ of rs3918249 MMP9 decreases risk for XFG $(\mathrm{OR}=0.75)$, and allele $\mathrm{G}$ of the $\mathrm{rs} 2250889$ $M M P 9$ locus increases the risk for the disease $(\mathrm{OR}=1.59$ 1.68) in the Caucasian population of Central Russia. These loci and 12 SNPs linked to them appear to have significant regulatory potential and may affect the expression of 13 genes and alternative splicing of 4 genes in various tissues and organs related to the pathogenesis of XFG. The obtained results warrant further studies on the possible contribution of the $M M P$ genes to various eye disorders.

\section{Statement of Ethics}

This study was approved by the Ethics Committee of Belgorod State University (Protocol No. 4, April 8, 2015). All study participants signed written informed consent per the principles of the Helsinki Declaration before enrollment in the study.

\section{Conflict of Interest Statement}

The authors have no conflicts of interest to declare.

\section{Funding Sources}

This is a self-funded work with no external sponsorship.

\section{Author Contributions}

D.S., V.D., and M.C.: substantial contributions to conception and design. D.S. and E.R.: acquisition of data. I.P. and V.D.: analysis and interpretation of data. D.S. and I.P.: drafting the article. E.R., V.D., and M.C.: revising it critically for important intellectual content. All authors gave final approval of the version to be published. All authors are accountable for all aspects of the work in ensuring that questions related to the accuracy or integrity of any part of the work are appropriately investigated and resolved. 


\section{References}

1 Quigley HA, Broman AT. The number of people with glaucoma worldwide in 2010 and 2020. Br J Ophthalmol. 2006;90(3):262-7.

2 European Glaucoma Society. Terminology and guidelines for glaucoma. 4th ed. Savona, Italy: Publicomm Press; 2014.

3 Heijl A, Bengtsson B, Hyman L, Leske MC. Natural history of open-angle glaucoma. Ophthalmology. 2009;116(12):2271-6.

4 Ritch R, Schlötzer-Schrehardt U, Konstas AG. Why is glaucoma associated with exfoliation syndrome? Prog Retin Eye Res. 2003;22(3): 253-75.

5 Schlötzer-Schrehardt U, Naumann GO. Ocular and systemic pseudoexfoliation syndrome. Am J Ophthalmol. 2006;141(5):921-37.

6 Rasmussen CA, Kaufman PL. The trabecular meshwork in normal eyes and in exfoliation glaucoma. J Glaucoma. 2014;23(8 Suppl 1): S15-9.

7 Cui N, Hu M, Khalil RA. Biochemical and biological attributes of matrix metalloproteinases. Prog Mol Biol Transl Sci. 2017;147:1-73.

8 Maatta M, Tervahartiala T, Harju M, Airaksinen J. Matrix metalloproteinases and their tissue inhibitors in aqueous humor of patients with primary open-angle glaucoma, exfoliation syndrome, and exfoliation glaucoma. J Glaucoma. 2005;14:64-9.

9 Singh M, Tyagi SC. Metalloproteinases as mediators of inflammation and the eyes: molecular genetic underpinnings governing ocular pathophysiology. Int J Ophthalmol. 2017; 10(8):1308-18

10 Schlotzer-Schrehardt U, Lommatzsch J, Kuchle M, Konstas A, Naumann G. Matrix metalloproteinases and their inhibitors in aqueous humor of patients with pseudoexfoliation syndrome/glaucoma and primary open-angle glaucoma. Invest Ophthalmol Vis Sci. 2003;44:1117-25.

11 Agapova OA, Ricard CS, Salvador-Silva M, Hernandez MR. Expression of matrix metalloproteinases and tissue inhibitors of metalloproteinases in human optic nerve head astrocytes. Glia. 2001;33(3):205-16.

12 Agapova OA, Kaufman PL, Lucarelli MJ, Gabelt BT, Hernandez MR. Differential expression of matrix metalloproteinases in monkey eyes with experimental glaucoma or optic nerve transection. Brain Res. 2003; 967(1-2):132-43.
13 Chintala SK, Zhang X, Austin JS, Fini ME. Deficiency in matrix metalloproteinase gelatinase B (MMP-9) protects against retinal ganglion cell death after optic nerve ligation. J Biol Chem. 2002;277(49):47461-8.

14 Tsironi EE, Pefkianaki M, Tsezou A, Kotoula MG, Dardiotis E, Almpanidou P, et al. Evaluation of MMP1 and MMP3 gene polymorphisms in exfoliation syndrome and exfoliation glaucoma. Mol Vis. 2009;15:2890-5.

15 Mossböck G, Weger M, Faschinger C, Zimmermann C, Schmut O, Renner W, et al. Role of functional single nucleotide polymorphisms of MMP1, MMP2, and MMP9 in open angle glaucomas. Mol Vis. 2010;16:1764-70.

16 Wu MY, Wu Y, Zhang Y, Liu CY, Deng CY, Peng L, et al. Associations between matrix metalloproteinase gene polymorphisms and glaucoma susceptibility: a meta-analysis. BMC Ophthalmol. 2017;17(1):48.

17 He M, Wang W, Han X, Huang W. Matrix metalloproteinase-1 rs1799750 polymorphism and glaucoma: a meta-analysis. Ophthalmic Genet. 2017;38(3):211-6.

18 Eliseeva NV. A replicative study of the associations of polymorphic loci of the LOXL1 and GDKN2B-AS1 genes with the development of primary open-angle glaucoma in men of the Central Black Earth Region of the Russian Federation. Research Results in Biomedicine. 2020;6(2):198-208

19 Ponomarenko I, Reshetnikov E, Altuchova O, Polonikov A, Sorokina I, Yermachenko A, et al. Association of genetic polymorphisms with age at menarche in Russian women. Gene. 2019;686:228-36.

20 Svinareva DI. The contribution of gene-gene interactions of polymorphic loci of matrix metalloproteinases to susceptibility to primary open-angle glaucoma in men. Research Results in Biomedicine. 2020;6(1):63-77.

21 Kingwell BA, Medley TL, Waddell TK, Cole TJ, Dart AM, Jennings GL. Large artery stiffness: structural and genetic aspects. Clin Exp Pharmacol Physiol. 2001;28(12):1040-3.

22 Taylor J, Sun YV, Chu J, Mosley TH, Kardia SL. Interactions between metallopeptidase 3 polymorphism rs679620 and BMI in predicting blood pressure in African-American women with hypertension. J Hypertens. 2008; 26(12):2312-8

23 Che R, Jack JR, Motsinger-Reif AA, Brown CC. An adaptive permutation approach for genome-wide association study: evaluation and recommendations for use. BioData Min. 2014;7:9.
24 Ward LD. Kellis M HaploReg: a resource for exploring chromatin states, conservation, and regulatory motif alterations within sets of genetically linked variants. Nucleic Acids Res. 2012;40:D930-4.

25 Guo L, Moss SE, Alexander RA, Ali RR, Fitzke FW, Cordeiro MF. Retinal ganglion cell apoptosis in glaucoma is related to intraocular pressure and IOP-induced effects on extracellular matrix. Invest Ophthalmol Vis Sci. 2005; 46(1):175-82.

26 Ritch R, Schlötzer-Schrehardt U. Exfoliation syndrome. Surv Ophthalmol. 2001;45(4): 265-315.

27 Gartaganis SP, Georgakopoulos CD, Mela EK, Exarchou A, Ziouti N, Assouti M, et al. Matrix metalloproteinases and their inhibitors in exfoliation syndrome. Ophthalmic Res. 2002;34(3):165-71.

28 Awadalla MS, Burdon KP, Kuot A, Hewitt AW, Craig JE. Matrix metalloproteinase-9 genetic variation and primary angle closure glaucoma in a Caucasian population. Mol Vis. 2011;17:1420-4.

29 Shi H, Zhu R, Hu N, Shi J, Zhang J, Jiang L, et al. Association of frizzled-related protein (MFRP) and heat shock protein 70 (HSP70) single nucleotide polymorphisms with primary angle closure in a Han Chinese population: Jiangsu Eye Study. Mol Vis. 2013;19:128-34.

30 Gao XJ, Hou SP, Li PH. The association between matrix metalloprotease- 9 gene polymorphisms and primary angle-closure glaucoma in a Chinese Han population. Int J Ophthalmol. 2014;7(3):397-402.

31 Chen X, Chen Y, Wiggs JL, Pasquale LR, Sun X, Fan BJ. Association of matrix metalloproteinase-9 (MMP9) variants with primary angle closure and primary angle closure glaucoma. PLoS One. 2016;11(6):e0157093.

32 Zhao F, Fan Z, Huang X. Role of matrix metalloproteinase- 9 gene polymorphisms in glaucoma: a hospital-based study in Chinese patients. J Clin Lab Anal. 2020;34(3):e23105.

33 Zhang Y, Wang M, Zhang S. Association of MMP-9 gene polymorphisms with glaucoma: a meta-analysis. Ophthalmic Res. 2016;55(4): 172-9.

34 Cong Y, Guo X, Liu X, Cao D, Jia X, Xiao X, et al. Association of the single nucleotide polymorphisms in the extracellular matrix metalloprotease-9 gene with PACG in southern China. Mol Vis. 2009;15:1412-7. 